\title{
Improving referrals for tilt table testing in patients with transient loss of consciousness
}

\author{
Luke Thornton, Nithusa Rahunathan, Narain Verma, Kenneth Wong
}

Castle Hill Hospital, Hull, UK

\begin{abstract}
Tilt table testing has been used for over twenty years in the investigation of patients with transient loss of consciousness. The European Society of Cardiology (ESC) recently recommended new guidance regarding indications for tilt table testing. We conducted an educational intervention and produced a new referral proforma that referring clinicians are expected to fill in for all patients referred for tilt table testing. At baseline, $76 \%$ ( $n=84)$ of referrals for tilt table testing were made in accordance to ESC guidance.

Following a simple educational intervention, $100 \%(n=6)$ were in line with ESC guidance. After the introduction of the referral proforma, 92\% $(n=12)$ of referrals followed ESC guidance. At final data collection, $100 \%(n=11)$ of referrals followed ESC guidance. In conclusion, a simple educational intervention and the use of a referral proforma in this quality improvement project have made a sustained difference in improving the appropriateness of referrals for tilt table testing. This has the potential to optimise the efficient use of resources and improve patient care through avoiding unnecessary investigation.
\end{abstract}

\section{Problem}

Transient loss of consciousness (TLOC) is a common problem and the diagnosis is often challenging. After careful history taking, physical examination and basic tests, the diagnosis is clear in many patients. But a significant proportion of patients with syncope require further investigations to characterize the cause of their TLOC. This quality improvement project aimed to improve the standard of referrals received from Castle Hill Hospital in Hull for tilt table testing in patients with TLOC.

Tilt table testing has been performed at Castle Hill hospital since mid-2011. Currently, approximately five patients are referred for testing each month with one to three tests being performed each week excluding public holidays. Not all of these referrals derive from Castle Hill Hospital as several hospitals in the region also refer their patients for tilt table testing at the tertiary centre.

In a departmental audit of 217 consecutive patients undergoing tilt testing, only one patient had cardioinhibitory response, 20 had vasodepressor response, three had mixed response, and $15 \mathrm{had}$ postural orthostatic tachycardia syndrome (POTS). Eighty-two percent had normal tests. In order to optimise the use of resources to improve the diagnostic yield, we aimed to find out whether patients are referred according to guidance from the European Society of Cardiology (ESC).[1]

While improving the appropriateness of referrals was our aim, our project also has the potential to improve patient care through avoiding unnecessary investigations, further improving the efficiency of the service and reducing cost.

Our project team consisted of one F1 doctor (Luke Thornton), two medical students (Narain Verma; Nithusa Rahunathan), and one consultant cardiologist (Kenneth Wong).

\section{Background}

Tilt table testing has been used for over twenty years in the investigation of patients with TLOC. The key aim is to ensure patients with cardioinhibitory or mixed responses are diagnosed early since they might benefit from permanent pacemaker implantation. Furthermore, postural orthostatic tachycardia syndrome (POTS) can also be diagnosed by tilt testing.[2]

The ESC recently recommended new guidance regarding indications for tilt table testing:

Class I indication - one episode of unexplained syncope in high risk setting OR recurrent episodes in the absence of heart disease or in the presence of organic heart disease, after cardiac causes of syncope have been excluded OR to demonstrate susceptibility to reflex syncope to the patient.

Class Ila - to discriminate between reflex and orthostatic syncope.

Class Illb - to discriminate between syncope and epilepsy OR a patient with recurrent unexplained falls $O R$ frequent syncope in patients with psychiatric disease. These indications correlate with the level of evidence base: class I indication has evidence that a given treatment or procedure is beneficial, while evidence for class Ilb indication is less well established.[1]

\section{Baseline measurement}

We retrospectively audited 120 consecutive patients who had undergone tilt table testing at our institution. Data were collected 
using the hospital database and reports produced after each patient had undergone testing. Ten patients were excluded from analysis due to missing information.

Seventy-six percent of referrals were deemed appropriate in accordance with ESC guidance $(n=84): 60 \%(n=66)$ Class I; $4 \%$ $(n=4)$ Class $2 a ; 2 \%(n=2)$ Class $2 b ; 11 \%(n=12)$ POTS. However $24 \%(n=26)$ did not have an appropriate referral indication in line with ESC guidance or POTS.

See supplementary file: ds6984.jpg - "Referral Proforma"

\section{Design}

Following baseline measurement, it was clear that the standard of referrals at our institution for tilt table testing could be improved. We felt that a departmental educational intervention in the form of a lecture from a consultant cardiologist was the most appropriate initial intervention. We felt this was low cost, simple to undertake, and should improve referrals of all referring doctors.

We also considered the possible implementation of a referral proforma depending on results following the educational intervention. This would be available in the outpatient department and should be filled in by clinicians referring patients for tilt table testing.

The project was initiated in early February 2015. The departmental educational intervention was delivered on the 17th February 2015. Repeat measurements were initially carried out after one month, although this was increased to three months following the small number of patients undergoing testing following PDSA cycle 1. Measurements were concluded in October 2015

\section{Strategy}

PDSA cycle 1: It was decided that an educational intervention was appropriate in order to improve the standard of referrals for tilt table testing. The educational intervention was in the form of a lecture to medical trainees with a focus on ESC guidance for tilt testing. This was delivered on the 17th February 2015.

PDSA cycle 2: In addition to this, a referral proforma was designed for all patients referred for tilt testing. The new referral proforma was presented at the cardiology department audit meeting on the 20th March 2015, attended by more senior doctors/consultant cardiologists.

Final measurement: An additional round of data collection was performed on the 8th October 2015 to ensure sustained improvement in the standard of referrals. No further changes were made at this stage due to marked improvement in referral standards.

\section{Results}

PDSA cycle 1: Six out of six patients were referred appropriately
(100\%) - five were referred in accordance to ESC guidance Class I indication and one had Class $2 b$ indication (table 1 ).

PDSA cycle 2: 12 out of 13 patients were referred appropriately $(92 \%)$ - nine were referred in accordance to ESC guidance Class I indication, one had Class 2a indication and two had POTS (table 1).

Final measurement: 11 out of 11 patients were referred appropriately (100\%): 10 were referred in accordance to ESC guidance Class I indication and one had Class $2 \mathrm{a}$ indication, indicating sustained improvement compared with baseline (table 1).

See supplementary file: ds7236.png - "Table 1 - Results"

\section{Lessons and limitations}

A simple education intervention and the use of a referral proforma in this quality improvement project have made a sustained difference in improving the appropriateness of referrals for tilt table testing. The total number of appropriate referrals increased from $76 \%$ at baseline to $92-100 \%$.

The use of a referral proforma also helps us triage referrals so those patients with Class I indications might be prioritised if there is a growing number of referrals, exceeding the capacity of the service.

Not all doctors were using the referral proforma since its introduction despite the publicity (PDSA cycle 2). The department still receives typed letters from clinics asking for tilt table tests. There is inherent delay in getting letters typed by secretaries, letters getting signed by consultants/registrars, and then posted to the department.

Other limitations include the small number of patients in PDSA cycles 1 and 2 and final measurement, although a large number of patients were studied at baseline. However, to avoid bias, we studied a consecutive cohort of NHS patients.

\section{Conclusion}

A simple educational intervention and the use of a referral proforma in this quality improvement project have made a marked difference in improving the appropriateness of referrals for tilt table testing. The improvement in quality appears to be sustained. Future quality improvement projects might focus on the introduction of an electronic referral proforma.

This has the potential to further improve efficiency of the referral system and enable more efficient re-auditing of the appropriateness of referrals, as well as timeliness of performance of the tilt table tests. We feel these improvements could be replicated through similar methodology in other NHS hospitals if required.

\section{References}

1. European Society of Cardiology (ESC). Guidelines for the 
diagnosis and management of syncope. Eur Heart $\mathrm{J}$ 2009;30:2631-71.

2. Soliman et. al. Postural orthostatic tachycardia syndrome (POTS): a diagnostic dilemma. Br J Cardiol 2010;17:36-9.

\section{Declaration of interests}

Nothing to declare.

\section{Acknowledgements}

loannis Kasouridis.

\section{Ethical approval}

Work was deemed an improvement study and not a study on human subjects, thus no ethical approval required. 